\title{
Kv3 Channels Contribute to the Excitability of Subpopulations of Spinal Cord Neurons in Lamina VII
}

\author{
Pierce N. Mullen, ${ }^{1}$ Nadia Pilati, ${ }^{2}$ Charles H. Large, ${ }^{3}$ Jim Deuchars, ${ }^{1}$ and Susan A. Deuchars ${ }^{1}$ \\ https://doi.org/10.1523/ENEURO.0510-21.2021 \\ ${ }^{1}$ School of Biomedical Sciences, Faculty of Biological Sciences, University of Leeds, Leeds, LS29JT United Kingdom, \\ ${ }^{2}$ Autifony Srl, 35127 Padua, Italy, and ${ }^{3}$ Autifony Therapeutics Ltd, Stevenage, SG12FX United Kingdom
}

\begin{abstract}
Autonomic parasympathetic preganglionic neurons (PGNs) drive contraction of the bladder during micturition but remain quiescent during bladder filling. This quiescence is postulated to be because of recurrent inhibition of PGN by fast-firing adjoining interneurons. Here, we defined four distinct neuronal types within Lamina VII, where PGN are situated, by combining whole cell patch clamp recordings with k-means clustering of a range of electrophysiological parameters. Additional morphologic analysis separated these neuronal classes into parasympathetic preganglionic populations (PGN) and a fast-firing interneuronal population. Kv3 channels are voltage-gated potassium channels $(\mathrm{Kv})$ that allow fast and precise firing of neurons. We found that blockade of Kv3 channels by tetraethylammonium (TEA) reduced neuronal firing frequency and isolated high-voltage-activated Kv currents in the fast-firing population but had no effect in PGN populations. Furthermore, Kv3 blockade potentiated the local and descending inhibitory inputs to PGN indicating that Kv3-expressing inhibitory neurons are synaptically connected to PGN. Taken together, our data reveal that Kv3 channels are crucial for fast and regulated neuronal output of a defined population that may be involved in intrinsic spinal bladder circuits that underpin recurrent inhibition of PGN.
\end{abstract}

Key words: bladder reflex; Kv3 channels; parasympathetic; spinal cord

\section{Significance Statement}

Neural circuits in the spinal cord and pons mediate the micturition reflex. The spinal cord drives bladder contraction during micturition through the activation of parasympathetic preganglionic neurons (PGNs) in Lamina VII of the sacral spinal cord. Despite the significant contribution of these neurons to a crucial physiological reflex, neurons in this region have been under-characterized. This study therefore elucidated and thoroughly characterized distinct neuronal populations in this lamina; we propose that these populations included a fast-firing interneuron and subtypes of PGNs. Further investigation revealed the critical importance of Kv3 channels in the fast-firing ability of the interneurons, as well as in synaptic release onto PGNs.

\section{Introduction}

Autonomic parasympathetic preganglionic neurons (PGNs) are situated at the intersection between the lumbar and sacral spinal cord in the intermediolateral laminae, and drive the contraction of the bladder detrusor muscle during the micturition reflex (de Groat and Ryall, 1968a; Morgan et al., 1979; Fowler et al., 2008; de Groat

Received December 13, 2021; accepted December 21, 2021; First published January 20, 2022.

The authors declare no competing financial interests. et al., 2015). The quiescence of PGN during bladder filling has been postulated to be due to recurrent inhibition mediated by parasympathetic axon collaterals and fastfiring interneurons in the vicinity of PGN (de Groat and Ryall, 1968b; Shefchyk, 2001). A description of neurons in this area and the mechanisms that confer the fast-firing

\footnotetext{
Author contributions: P.N.M., N.P., C.H.L., J.D., and S.A.D. designed research; P.M. performed research; N.P. and C.H.L. contributed unpublished reagents/analytic tools; P.N.M., N.P., J.D., and S.A.D. analyzed data; P.N.M., J.D., and S.A.D. wrote the paper.
} 
phenotype is lacking, but one strong possibility for a biophysical mechanism promoting fast firing is the expression of Kv3 channels.

Kv3 voltage-gated potassium channels play an important role in shaping a fast-firing phenotype and precise synaptic output of neurons (Rudy and McBain, 2001). This role is conferred by two important properties; channel activation at relatively high voltages only achieved during an action potential (AP), and fast activation and deactivation kinetics that ensure a rapid repolarization of the membrane and a short refractory period. Rapid repolarization produces brief APs, which limit calcium influx and thus neurotransmitter release at the presynaptic terminal, whereas short refractory periods, in addition to brief APs, allow the neuron to fire APs in quick succession (Kaczmarek and Zhang, 2017). These channels, therefore, are crucial in determining the ability of a neuron to produce a fast but precise output.

Kv3 channels are typically expressed in the soma, nodes of Ranvier and synaptic terminals of neurons in various regions of the CNS, from the auditory brainstem to the cortex (Weiser et al., 1995). Expression has also been observed in the thoracic spinal cord, particularly in dorsal horn interneurons, Renshaw cells and intermediolateral laminae typically associated with sympathetic preganglionic neurons (Deuchars et al., 2001; Brooke et al., 2002, 2006; Song et al., 2006; Nowak et al., 2011).

However, the connection between parasympathetic preganglionic output from the spinal cord and Kv3-mediated fast firing of neurons in the vicinity of PGN has not been established. We thus hypothesized that Kv3 channels are indeed expressed in putative interneurons in the autonomic intermediolateral lamina of the lumbosacral spinal cord and are responsible for facilitating a fast-firing phenotype, making them ideal candidates for autonomic inhibition.

\section{Materials and Methods}

\section{Spinal cord tissue preparation}

C57Bl6 mice (postnatal day 10-21), according with the United Kingdom animals (Scientific Procedures) Act 1986 and ethical standards set out by the University of Leeds Ethical Review Committee, were anaesthetized by intraperitoneal injection of sodium pentobarbitone (Euthanal, $60 \mathrm{mg} / \mathrm{kg}$ ). Upon complete loss of pedal withdrawal, a transverse laparotomy was conducted to remove the ventral ribs and expose the heart. The right atrium was incised and $20 \mathrm{ml}$ of artificial CSF (aCSF) with high sucrose

\footnotetext{
This work was supported by the Biotechnology and Biological Sciences Research Council Grant BB/L01565X/1. LSM880 + Airyscan confocal microscope was supported by Wellcome Trust WT104918MA

P. Mullen's present address: School of Psychology and Neuroscience, University of St Andrews, St Andrews, KY169JP, Scotland.

Correspondence should be addressed to Susan Deuchars at s.a. deuchars@leeds.ac.uk or Pierce Mullen at pnm1@st-andrews.ac.uk.

https://doi.org/10.1523/ENEURO.0510-21.2021 Copyright (C) 2022 Mullen et al.

This is an open-access article distributed under the terms of the Creative Commons Attribution 4.0 International license, which permits unrestricted use, distribution and reproduction in any medium provided that the original work is properly attributed.
}

(sucrose aCSF; $217 \mathrm{~mm}$ sucrose, $2 \mathrm{~mm} \mathrm{NaHCO} 3,3 \mathrm{~mm}$ $\mathrm{KCl}, 2 \mathrm{~mm} \mathrm{MgSO} 4.7 \mathrm{H}_{2} \mathrm{O}, 2.5 \mathrm{~mm} \mathrm{NaH}_{2} \mathrm{PO}_{4}, 10 \mathrm{~mm}$ glucose, and $\left.1 \mathrm{~mm} \mathrm{CaCl}_{2}\right)$ oxygenated $\left(95 \% \mathrm{O}_{2} / 5 \% \mathrm{CO}_{2}\right)$ on ice was perfused through the left ventricle and into the circulation system. The mouse was decapitated, the skin removed, and a dorsal laminectomy conducted to expose the spinal cord which was removed following cutting of the rootlets attached to the cord.

Upon removal, the spinal cord was placed in a Petridish containing ice-cold sucrose aCSF under a dissecting microscope (SM2 2B, Nikon) and the meninges that ensheathe the cord were removed with fine forceps. Lumbosacral segments of the spinal cord were set in 3\% agar in aCSF (124 mм NaCl, $26 \mathrm{~mm} \mathrm{NaHCO} 3,3 \mathrm{~mm} \mathrm{KCl,} 2 \mathrm{~mm}$ $\mathrm{MgSO}_{4} .7 \mathrm{H}_{2} \mathrm{O}, 2.5 \mathrm{~mm} \mathrm{NaH} \mathrm{PO}_{4}, 10 \mathrm{~mm}$ glucose, and $2 \mathrm{mM} \mathrm{CaCl}_{2}$ ), mounted against a $4 \%$ block of agar for stability using superglue and sectioned in a bath of oxygenated ice-cold sucrose aCSF using an Integraslice 7550 PSDS (Campden Instruments) microtome. Transverse sections were cut at $250-400 \mu \mathrm{m}$ and incubated in an oxygenated holding chamber containing aCSF and allowed to recover for $1 \mathrm{~h}$ before recording.

\section{Patch clamp recordings of spinal neurons}

Recordings were conducted at $34^{\circ} \mathrm{C}$ using an inline solution heater and temperature controller (SH27B, TC344C, Warner Instruments). Slices were transferred to and immobilized in an incubation chamber perfused with oxygenated aCSF at a rate of $3-5 \mathrm{ml} / \mathrm{min}$. Thick-walled borosilicate glass microelectrodes (inner diameter 0.86 $\mathrm{mm}$, outer diameter $1.5 \mathrm{~mm}$ ) were fabricated using a Sutter P97 micropipette puller (Sutter Instruments) with resistances of 5-9 $\mathrm{M} \Omega$. The recording patch and bath electrodes used a silver/silver chloride $\left(\mathrm{Ag} / \mathrm{AgCl}_{2}\right)$ interface. Patch microelectrodes were filled with an intracellular solution composed of the following: $110 \mathrm{~mm} \mathrm{~K}$ gluconate, $11 \mathrm{~mm}$ EGTA, $2 \mathrm{~mm} \mathrm{MgCl}_{2}, 0.1 \mathrm{~mm} \mathrm{CaCl}_{2}, 10$ mm HEPES, $2 \mathrm{~mm} \mathrm{Na}{ }_{2}$ ATP, $0.3 \mathrm{~mm} \mathrm{Na} \mathrm{NaTP}_{2}$ and $0.5 \%$ Neurobiotin (Vector Laboratories), pH 7.4, 290 mOsm. Recordings were obtained at $50 \mathrm{kHz}$, filtered through a Bessel low-pass filter at $10 \mathrm{kHz}$ using a MultiClamp $700 \mathrm{~A}$ (Molecular Devices), digitized using a Digidata 1322A (Molecular Devices) and recorded in pClamp9 software. An upright microscope (Olympus BX50W1), camera (QImaging Rolera-XR, QImaging), and QCapture software (QImaging) were used to visualize the spinal cord section and center the stage over the region of interest (ROI), e.g., the lateral region of the lumbo-sacral spinal cord sections.

In current clamp configuration, neurons were characterized by long ( $1 \mathrm{~s}$ ) hyperpolarizing and depolarizing current steps from a holding membrane potential of $-70 \mathrm{mV}$. The holding membrane potential was corrected for a liquid junction potential of $-15 \mathrm{mV}$. Passive properties such as cell capacitance and resistance were monitored with a 50-pA hyperpolarizing pulse at the end of each sweep by fitting an exponential function to the voltage decay. Neuronal firing frequency was calculated during 1-s current injections, incrementally increasing by $10 \mathrm{pA}$. The following frequency of neuronal firing was assessed by applying trains of 10-ms square current pulses to neurons 
Table 1: Antibodies and concentrations used

\begin{tabular}{|c|c|c|c|c|c|c|}
\hline Target & Supplier & $\begin{array}{l}\text { Species } \\
\text { raised in }\end{array}$ & Dilution & Secondary detection & $\begin{array}{l}\text { Catalog } \\
\text { number }\end{array}$ & Reference \\
\hline $\begin{array}{l}\text { GlyT2 (glycine } \\
\text { transporter) }\end{array}$ & $\begin{array}{l}\text { Synaptic } \\
\text { Systems }\end{array}$ & Rabbit & $1: 2000$ & $\begin{array}{l}\text { Donkey } \alpha \text {-Rabbit Alexa } 488 \text { (Life Technologies, } \\
\text { A21206) }\end{array}$ & 272003 & $\begin{array}{l}\text { Jursky and } \\
\text { Nelson } \\
\text { (1995) }\end{array}$ \\
\hline $\begin{array}{l}\text { VGluT2 (vesic- } \\
\text { ular gluta- } \\
\text { mate } \\
\text { transporter) }\end{array}$ & $\begin{array}{l}\text { Synaptic } \\
\text { Systems }\end{array}$ & Rabbit & $1: 2000$ & $\begin{array}{l}\text { Donkey } \alpha \text {-Rabbit Alexa } 488 \text { (Life Technologies, } \\
\text { A21206) }\end{array}$ & 135403 & $\begin{array}{l}\text { Zhu et al. } \\
\text { (2018) }\end{array}$ \\
\hline
\end{tabular}

at 20,50 , and $100 \mathrm{~Hz}$ at increasing current amplitudes. The rate of failure (\%) to evoke an AP at each frequency and current pulse was quantified.

In voltage clamp configuration, outward plateau and tail currents were measured during $250 \mathrm{~ms}, 10-\mathrm{mV}$ incrementing steps from $-56 \mathrm{mV}$. All voltages were corrected for the liquid junction potential and series resistance and holding currents were $<30 \mathrm{M} \Omega$ and $<300 \mathrm{pA}$. Recordings where series resistance changed by $>20 \%$ were excluded. Noninactivating Kv currents were isolated by inactivating channels with a 2-s voltage step to $-36 \mathrm{mV}$ before applying test voltage steps.

Postsynaptic currents were evoked by a brief pulse (6$8 \mathrm{~V}$ ) using a bipolar electrode positioned in the lateral white matter where descending tracts are located. EPSCs and IPSCs were recorded by holding the neuron at $-56 \mathrm{mV}$ for EPSCs and at $0 \mathrm{mV}$ for IPSCs. Paired pulse stimulation with an interpulse duration of $100 \mathrm{~ms}$ was used to assess presynaptic potentiation and attenuation. A $10-\mathrm{Hz}$ train of stimulation was also used to assess the synaptic response to repetitive input.

Recordings in control aCSF were started 4 min after achieving the whole cell configuration. Tetraethylammonium chloride (TEA) and dendrotoxin (DTX) were dissolved in extracellular solution to obtain a bath concentration of $0.5 \mathrm{~mm}$ and $10 \mathrm{~nm}$, respectively. Recordings were obtained after $8 \mathrm{~min}$ of dialysis for DTX experiments and after $4 \mathrm{~min}$ of dialysis for TEA experiments. Passive properties were monitored throughout and recordings where series resistance changed by $>20 \%$ were excluded.

\section{Morphologic reconstruction}

Recorded neurons were filled with $0.5 \%$ Neurobiotin and spinal cord slices were fixed on slides with $4 \%$ paraformaldehyde (PFA) for $2 \mathrm{~h}$. Fixed sections were then washed, permeabilized and incubated in Steptadavidin555 solution [1:1000, phosphate buffer (PB), 0.3\% Triton $\mathrm{X}$-100]. Sections were imaged using a Zeiss LSM880 Upright confocal microscope at $20 \times$ and traced using the
SimpleNeuriteTracer plugin in ImageJ. Sholl analysis was performed with the plugin and a custom python script was used to compute the angles between endpoints of neurites and the soma.

\section{Immunohistochemistry}

Female wild-type C57BL/6 mice (three-month-old) were anesthetized with intraperitoneal pentobarbitone sodium (Sagatal, $60 \mathrm{mg} / \mathrm{kg}$ ) and perfused transcardially with $4 \%$ PFA in 0.1 M PB, pH 7.4. After fixation, spinal levels L1, L6, $\mathrm{S} 1$ were dissected and incubated in $0.1 \mathrm{M}$ PBS containing $30 \%$ sucrose until the tissue sank to the bottom before being embedded and frozen in Surgipath FSC 22 Clear Frozen Section Compound (Leica) freezing medium on dry ice; $20-\mu \mathrm{m}$ sections were cut using a Leica CM1850 cryostat cooled to approximately $-15^{\circ} \mathrm{C}$ and mounted onto Superfrost plus (Menzel-Glaser, Thermo Scientific) slides. Sections were washed three times in PBS, incubated in 10 $\mathrm{mm}$ sodium citrate at $80^{\circ} \mathrm{C}$ for $20 \mathrm{~min}$ for antigen retrieval, washed a further three times in PBS and blocked and permeabilized for $1 \mathrm{~h}$ in $5 \%$ goat and donkey serum in $\mathrm{PB}$ (0.3\% Triton $\mathrm{X}-100)$. All primary antibodies were incubated overnight in PB (0.3\% Triton X-100), washed in PBS and incubated for $1 \mathrm{~h}$ for directly conjugated secondary antibodies, for $2 \mathrm{~h}$ for biotinylated secondary antibodies and for $30 \mathrm{~min}$ for streptavidin to avoid endogenous biotin labeling. Antibodies used are listed in Table 1.

\section{Confocal and Airyscan imaging}

Images of neurons were acquired with the nucleus in view. Differential interference contrast (DIC) was used to identify the cell outline and determine a visible nucleus while super-resolution Airyscan images were taken of the neurons with the Alexa 555 and Alexa 488 fluorophores being stimulated separately and emissions collected with appropriate bandpass filters for the fluorophores. At least nine neurons from three sections at L6-S1 were measured per animal $(n=3)$. The neuron perimeter was traced in ImageJ to create a ROI. A 3- $\mu \mathrm{m}$ band around the cell was 


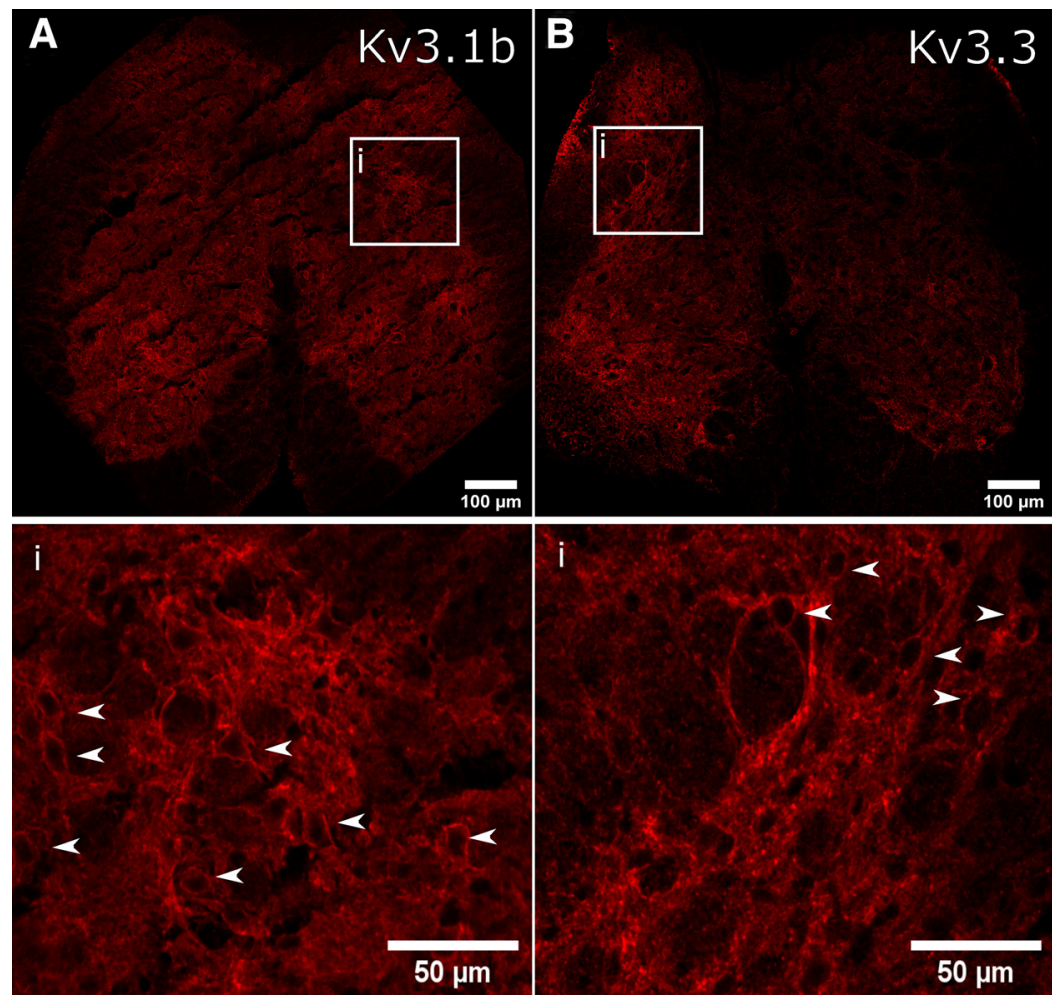

Figure 1. Immunofluorescent localization of Kv3 subunits in intermediolateral lumbosacral spinal cord. $\boldsymbol{A}, \boldsymbol{B}$, Immunofluorescence of Kv3.1b and Kv3.3 subunits. The white box outlines the intermediolateral autonomic area of the lumbosacral spinal cord and the bottom panels represents magnified images within this region. White arrows indicate Kv3 subunit immunofluorescence that appeared to encircle cells.

created from $2 \mu \mathrm{m}$ outside the perimeter to $1 \mu \mathrm{m}$ inside the perimeter. This formed a ROI from which synaptic immunoreactivity and Kv3 puncta in very close apposition with the cell membrane could be segmented. Synaptic immunoreactivity, referred to as boutons herein, and Kv3 puncta were segmented in ImageJ. For object-based co-localization, colocalization was defined as the center of a Kv3 punctum coinciding with the area of a bouton. This was performed using the JaCOP plugin (Bolte and Cordelieres (2006)) on Image J and reported as the percentage of co-localized boutons and as the percentage of co-localized puncta.

\section{Clustering of neuronal e-types}

Neurons were clustered based on seven features of neuron firing. These were frequency of APs, adaptation index, regularity of firing (interspike interval coefficient of variation), accommodation, as well as the presence of bursts, pauses or delays in firing. Ninety-six neurons from 35 mice were clustered on these features, after normalization, using SciPy K-means with the ideal number of clusters determined by an elbow plot. Principal component analysis (PCA) was performed using the SciPy package and used to reduce the electrophysiological parameter dimensions to visualize the k-mean clusters.

\section{Experimental design and statistical analysis}

Custom Python scripts were used to extract and measure APs, synaptic currents and outward currents. All statistics were conducted on GraphPad prism software with single neurons representing the experimental unit. ShapiroWilks and Levene's tested normality and homogeneity of variance assumptions. Kolmogorov-Smirnov test was used to statistically compare synaptic amplitude distributions. All statistical tests used are described alongside significant results. Values are given as mean \pm SD. Table 3 describes the tests used.

\section{Results}

\section{Kv3 subunits are expressed in the lumbosacral spinal cord}

Immuno-labeling of Kv3 subunits Kv3.1b and Kv3.3 revealed widespread expression in the lumbosacral spinal cord, throughout the dorsal, lateral and ventral gray matter, in agreement with previous descriptions of Kv3 subunits in other spinal segments (Brooke et al., 2002, 2006; Song et al., 2006; Nowak et al., 2011; Fig. 1A,B). Magnified images indicated a punctate expression profile within or closely apposed to the somatic and perisomatic membrane of neurons within the intermedio-lateral autonomic zone (Fig. 1B). Here, we define this region as within Lamina VII, lateral to the central canal and adjacent to the gray and white matter border.

\section{Identification of a fast-firing neuronal population, e-type 3, in the parasympathetic autonomic zone}

Neurons in the parasympathetic autonomic zone lack a robust characterization. We, therefore, performed an 
initial characterization based on the firing phenotype to understand the different neuronal types and identify putative Kv3-positive fast-firing cells in this area. Neurons were clustered according to a subset of electrophysiological characteristics such as firing frequency, adaptation index, inter spike interval coefficient of variation (synonymous with regularity), occurrence of delays, bursts, pauses, and accommodation of firing (Fig. 2A). Four distinct firing phenotypes, denoted e-types, were identified; e-type 1 steady regular, e-type 2 continuous adapting, e-type 3 fast bursting, e-type 4 steady delayed (Fig. 2C). E-type 3 had a markedly higher rate of firing than the other e-types, which were indistinguishable based on firing rate alone (Fig. 2B). E-type 3 had the briefest APs $(1.3 \pm 0.4 \mathrm{~ms})$ and largest AHP amplitude $(21.6 \pm 5.9 \mathrm{mV})$, classical indications of a contribution of Kv3 current (Table 2). Indeed, k-means clustering of AP waveforms for each cell revealed four AP-types. The briefest AP-type correlated most with the fast-firing e-type 3 (46\%), however AP waveform clustering did not fully overlap with previous e-type clusters defined by firing properties, suggesting waveform shape alone cannot be used to identify each neuronal type (Extended Data Fig. 2-1). In this initial characterization, we identified a fast-firing subpopulation and three slower neuronal phenotypes.

\section{Morphologic identification of PGN and interneurons}

In order to differentiate between putative PGN and interneurons, where possible because of full morphologic recovery, we assessed whether neuronal clusters had neurites projecting toward the ventral root, the site of efferent axonal exit from the spinal cord. This is an indicator of PGN (Morgan, 2002a). All e-types, except e-type 3, had neurons with clear ventral root-projecting (VRP) neurites (Fig. 3A). This is further represented by plotting the average distance and angle (in $45^{\circ}$ bins) of neurite end points from the soma for each e-type in the form of polar plots (Fig. 3C). E-types 1, 2, and 4 had long projections between $90^{\circ}$ and $135^{\circ}$, analogous to projecting toward the ventral root. Conversely, e-type 3 typically had a vertical orientation with short ventro-lateral projections and long dorsal projections. Kernel density estimates indicated the angular probability of projecting neurites; the peak angle for e-type 3 lay between $300^{\circ}$ and $360^{\circ}$ in the dorsal direction, whereas e-types 1 and 2 displayed prominent peaks in the lateral and dorso-medial direction, and e-type $4 \mathrm{had}$ similar occurrences in all directions (Fig. 3C). E-type morphologies could not be significantly distinguished by Sholl parameters, ramification index $(p=0.13)$ and critical value $(p=0.17$, one-way ANOVA; Fig. $3 B)$. However, this morphologic assessment indicated that e-type 3 lacked a ventral-root projection and thus likely did not belong to an autonomic preganglionic motoneuron class.

\section{Fast e-type 3 was able to follow high frequencies of stimuli}

To test the responsiveness of each phenotype to slow and fast stimuli we injected $10 \mathrm{~ms}$ square current pulses at varying frequencies $(20,50$, and $100 \mathrm{~Hz}$ ) and amplitudes $(160-400 \mathrm{pA})$ and quantified the rate of failure to evoke an AP for each pulse. Lower current amplitudes resulted in large failure rates as expected (Fig. 4A). E-type 3 had a significantly lower failure rate than the other e-types at 50 and $100 \mathrm{~Hz}$ when compared at a high current amplitude (350 pA, mixed effects ANOVA, $p=0.0421, p=0.005$, respectively). This indicated that e-type 3 was extremely responsive to high frequency stimulation (Fig. $4 A, B)$, a strong suggestion of functional expression of Kv3 channels.

\section{Fast e-type 3 firing was impaired by application of TEA}

We had uncovered a fast-firing cell type, e-type 3, within the autonomic intermediolateral laminae. Furthermore, e-type 3 also displayed features prototypical of Kv3 channel expression, namely fast and responsive firing and brief APs. We next began to address whether Kv3 channels were crucial to these properties by using low concentrations of tetraethylammonium (TEA; $0.5 \mathrm{~mm}$ ) to primarily block Kv3 channels (Fig. 5A, E1-4). Application of TEA significantly reduced firing frequency of e-type 3 by $24.5 \mathrm{~Hz}$ at $140 \mathrm{pA}$ (multiple paired $t$ tests with Bonferroni correction, $p=0.0095, n=4$, control; $60.25 \pm 16.98 \mathrm{~Hz}$, TEA; $35.75 \pm 14.97 \mathrm{~Hz}$; Fig. $5 A$, E3). TEA (0.5 mM) had no significant effect on other e-types. TEA can also block Kv1 channels, however, application of Kv1-specific blocker DTX (10 nм) had no discernible effect on firing frequency (Fig. 5B).

\section{A large proportion of e-type 3 outward current was TEA-sensitive}

Further interrogation with voltage clamp indicated low levels of DTX and TEA-sensitive current across all e-types (Fig. 6A,B). Subtraction of currents evoked in $0.5 \mathrm{~mm}$ TEA from control was used to isolate the TEA-sensitive Kv3 component of total Kv current (Fig. 6B). Interestingly, a large component of total Kv current was TEA and DTX insensitive, although this concentration of TEA does not produce a full block ( $50 \%)$ of $\mathrm{Kv} 3$ channels (Coetzee et al., 1999; Rudy and Mcbain, 2001). TEAsensitive current was significantly greater in e-type 3 at $14 \mathrm{mV}$ compared with e-type 2 and 4 (one-way ANOVA, $3760 \pm 1839$ vs $955 \pm 1012$ and $991.7 \pm$ $999.3 \mathrm{pA}, p=0.0116$ and $p=0.04$, respectively; Fig. $6 A)$. TEA-sensitive current contributed a small proportion of total Kv current across other e-types. Activation of TEA-sensitive current for e-type 3 neurons was shifted by $18 \mathrm{mV}$ toward more depolarized potentials compared with control (control V50 $=-28.18 \mathrm{mV}$, TEAsensitive V50 $-10.66 \mathrm{mV}$, curve fit comparison $p=$ 0.0012 , extra sum of squares $F$ test) suggesting that the TEA-sensitive current is produced by a high-voltage activated potassium channel, such as Kv3 channels. Here, through voltage clamp, we established that TEA-sensitive high-voltage activated putative Kv3 currents constituted a significant proportion of total $\mathrm{Kv}$ current in e-type 3 neurons and that it is most likely 


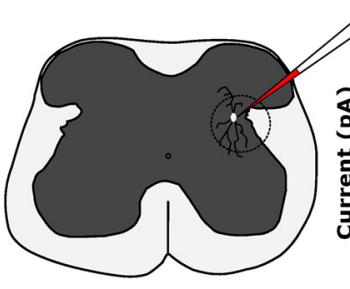

B

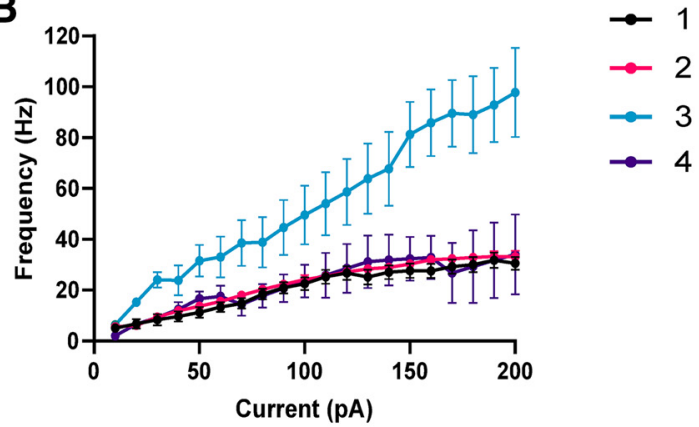

E-type 1

C Steady Regular

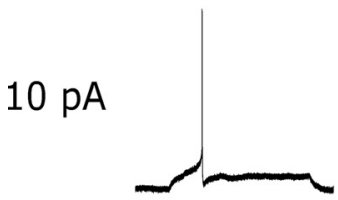

$50 \mathrm{pA} . \mid \mathrm{H}$
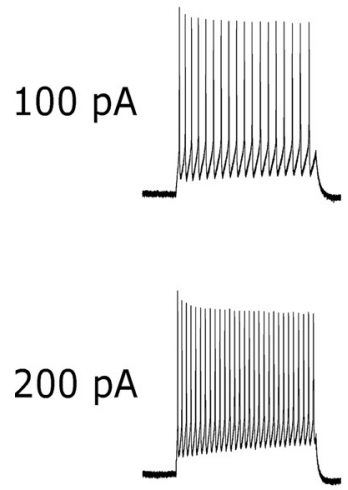

E-type 2

Continuous

adapting
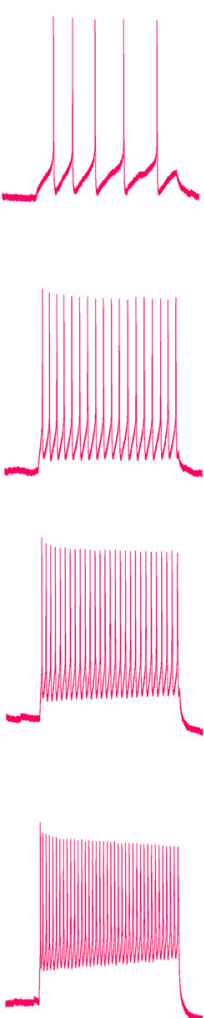

A
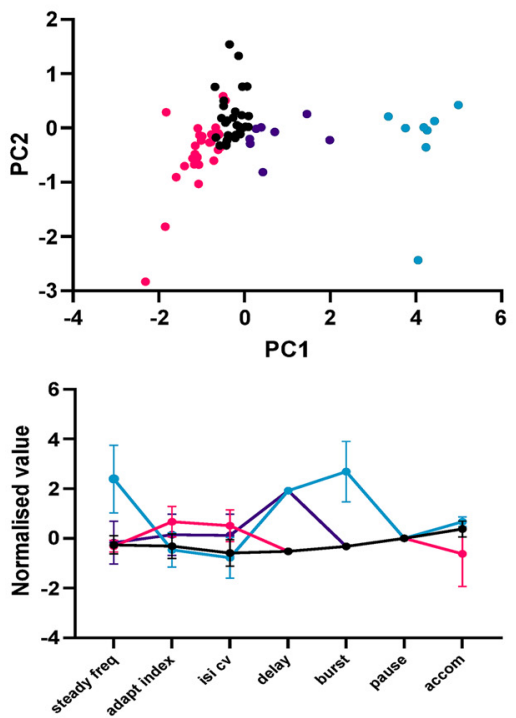

E-type 3

E-type 4

Fast Bursting

Steady

Delayed
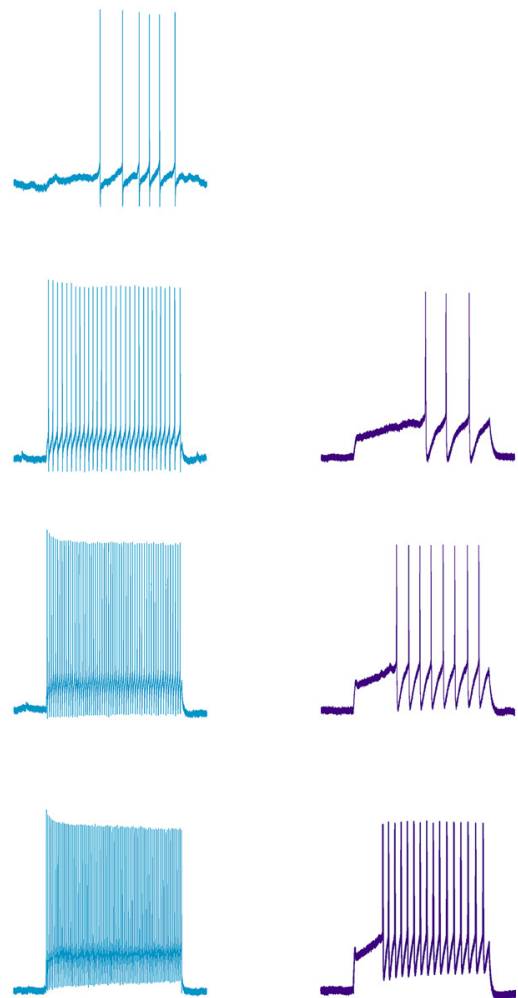

Figure 2. E-type classification of lumbosacral spinal neurons in Lamina VII. A, Neurons were clustered according to firing phenotype at maximal firing; steady state firing frequency, adaptation index, interspike interval coefficient of variation (ISI CV), delays, bursts, pauses, and accommodation were used as clustering variables. Upper panel, Principle component scatter plot summarizing clusters. Lower panel, Parallel plot comparison across clustering variables, error bars represent SEM. $\boldsymbol{B}$, Frequency-current plot summarizing the firing frequency elicited by 1-s current steps from $-70 \mathrm{mV}$ for different e-types, note the fast-firing e-type 3. C. Four different e-types: steady regular, continuous adapting, fast bursting, and steady delayed. Example traces for each e-type with 10, 50, 100, and 200 pA 1-s current injections. No firing phenotype was produced for e-type 4 at 10 pA. AP waveforms were also clustered to define AP-types and AP-types were correlated with e-types (Extended Data Fig. 2-1). PCA, principal component analysis; pA, picoAmps; mV, millivolts; Hz, Hertz; AP, Action potential. 
Table 2: Description of AP and afterhyperpolarization shape

\begin{tabular}{|c|c|c|c|c|}
\hline & E1 & E2 & E3 & E4 \\
\hline$\overline{\mathrm{AP}}$ width (ms) & $3.3 \pm 1.4$ & $4.0 \pm 1.8$ & $1.3 \pm 0.4$ & $3.5 \pm 2.0$ \\
\hline Repolarization duration (ms) & $2.4 \pm 1.1$ & $2.9 \pm 1.2$ & $0.8 \pm 0.3$ & $2.4 \pm 1.6$ \\
\hline AHP duration (ms) & $29.6 \pm 18.5$ & $28.8 \pm 16.3$ & $8.9 \pm 7.5$ & $27.1 \pm 20.6$ \\
\hline AHP amplitude (mV) & $19.5 \pm 5.3$ & $20.6 \pm 5.7$ & $21.6 \pm 5.9$ & $19.8 \pm 6.2$ \\
\hline
\end{tabular}

AP, Action potential; AHP, afterhyperpolarization; ms, milliseconds; mV, millivolts.

blockade of these channels with $0.5 \mathrm{~mm}$ TEA that reduced firing frequency.

\section{Local and descending inhibitory input to PGN was TEA-sensitive}

The data presented here provide strong evidence for the contribution of $\mathrm{Kv} 3$ channels to the rapid firing of a sub-population of neurons in the intermediolateral autonomic zone. However, Kv3 channels are also localized subcellularly and functionally important at the synaptic terminal, and likely to be important in any recurrent circuitry in the autonomic zone at the level of the synapse. To assess the role of Kv3 channels in synapses onto putative autonomic motoneurons in this region, such as etypes 1, 2, and 4, we evoked and recorded excitatory and inhibitory descending and local synaptic currents in the presence of TEA. A total of 13 out of 15 neurons belonged to the putative autonomic neuronal classes. Descending fibers containing predominantly excitatory (VGluT2) and some GABAergic and glycinergic descending bulbo-spinal input (Huma et al., 2014), were stimulated with a bipolar electrode placed in the lateral white matter of the lumbosacral spinal cord. Neurons were held at $0 \mathrm{mV}$ to record IPSCs and at $-56 \mathrm{mV}$ to record EPSCs. Application of $0.5 \mathrm{~mm}$ TEA increased evoked IPSC (eIPSC) amplitude by $>100 \%$ (paired $t$ test $p=0.03$, control $94.93 \pm$ 115.1 pA, TEA $210 \pm 134 \mathrm{pA}, n=9$; Fig. 7B). Paired-pulse stimulation was used to assess potential presynaptic site of action and TEA reduced the paired-pulse ratio (PPR) by $33 \%(p=0.0286, n=6$, control $1.564 \pm 0.59$, TEA $1.07 \pm$ 0.42 , paired $t$ test). Kv1 channels, also blocked by millimolar concentrations of TEA, are important regulators of synaptic excitability. To rule out a contribution to the observed effect we repeated the experiment in the presence of DTX (10 nm). DTX did not have a significant effect on

Table 3: Statistical table

\begin{tabular}{llll}
\hline $\begin{array}{l}\text { Data } \\
\text { structure }\end{array}$ & $N$ & Type of test & $\begin{array}{l}\text { Confidence } \\
\text { interval }\end{array}$ \\
\hline Normal & 6 & Paired $t$ test & -0.91 to -0.077 \\
Normal & 9 & Paired $t$ test & 11.88 to 218.6 \\
Normal & 11 & Paired $t$ test & -82.18 to -0.62 \\
Normal & $12,13,4,3$ & One-way ANOVA & -1.94 to 11.54 \\
Normal & $12,13,4,3$ & One-way ANOVA & -6 to 8 \\
Normal & $22,17,5$ & Mixed effects & 36.0 to 119.9 \\
Normal & $22,17,5$ & Mixed effects & 3.7 to 143.6 \\
Normal & 4 & Paired $t$ test & -37.59 to -11.41 \\
Normal & 8,3 & One-way ANOVA & -5007 to -602.9 \\
Normal & 3,3 & One-way ANOVA & 112.4 to 5423 \\
& & &
\end{tabular}

elPSC amplitude, suggesting eIPSC potentiation was solely because of Kv3 blockade (Fig. 7B). Super-resolution Airyscan co-immunolabelling of GlyT2 and VGAT confirmed the presence of inhibitory (glycinergic and GABAergic) synaptic terminal markers in the intermediolateral laminae of the lumbosacral spinal cord and revealed a degree of overlap with Kv3.1b (14\% and 13\%, respectively, of boutons positive for Kv3.1b; Fig. 7C). We delivered a train of stimuli to further demonstrate TEAsensitive potentiation of eIPSC amplitude during continuous activity. Histogram analysis of a $10-\mathrm{Hz}$ train of elPSCs demonstrated a significant shift toward larger amplitude elPSCs in the presence of TEA (two-sample Kolmogorov-Smirnov test, $p=0.0018$; Fig. 7D, left). Further analysis of spontaneous background IPSCs (sIPSC), likely because of local activity, also produced a similar shift toward larger sIPSC amplitudes during application of TEA (two-sample Kolmogorov-Smirnov test, $p=0.0004$; Fig. $7 D$, right). Most synaptic responses depressed over the course of the $10-\mathrm{Hz}$ train with a mean linear fit gradient of $-2.66 \pm 5.6$. Lack of activity-dependent facilitation here suggested that the fast-inactivating Kv3 subunit Kv3.4, was not functionally expressed at these synapses and was not the target of the effect of TEA. Together, these data strongly indicated that Kv3 channels were expressed in inhibitory terminals and functionally constrained both descending and local inhibitory synaptic amplitude.

\section{Local and descending excitatory input to PGN was TEA-insensitive}

Conversely, application of TEA had no significant effect on eEPSC amplitude and PPR (Fig. 8A). Interestingly, incubation in DTX significantly decreased eEPSC amplitude (control; $134.2 \pm 72.9 \mathrm{pA}$, DTX; $92.78 \pm 53.90 \mathrm{pA}$, paired $t$ test, $p=0.047$; Fig. $7 B$ ) and shifted cumulative frequency EPSC amplitude toward smaller amplitudes for evoked but not sEPSCs (two-sample Kolmogorov-Smirnov test, $p=$ 0.001; Fig. 8C). This implied that blocking Kv1 channels decreased synaptic amplitude only in descending excitatory input. Immuno-labeling revealed a degree of overlap between Kv3.1b and VGluT2 immunoreactivity (12\%; Fig. 8E), suggesting expression of Kv3 channels in a small proportion of excitatory synaptic terminals in this area.

The combined analyses presented here suggests that Kv3 channels are expressed in the somatic and synaptic membrane of inhibitory interneurons in the intermediolateral autonomic zone of the lumbosacral spinal cord, and that here they facilitate a distinctive fast-firing phenotype 
A

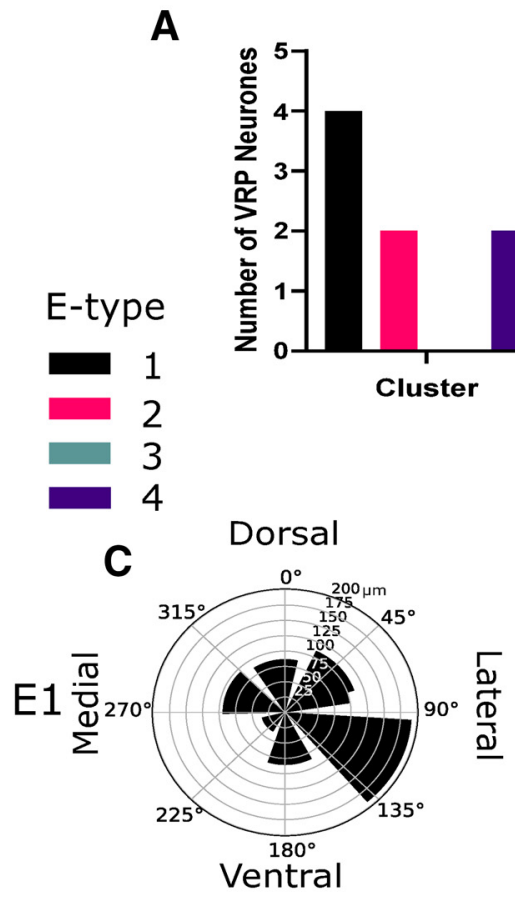

Dorsal
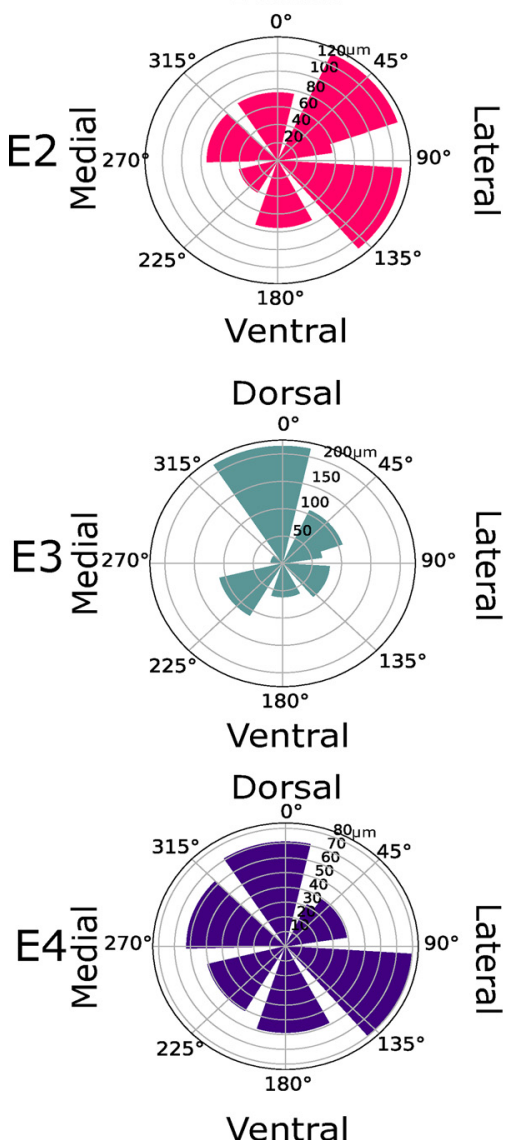

B

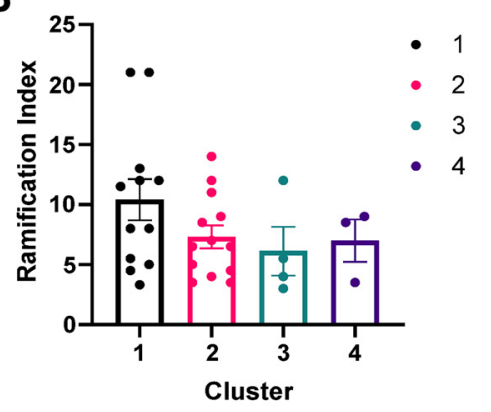

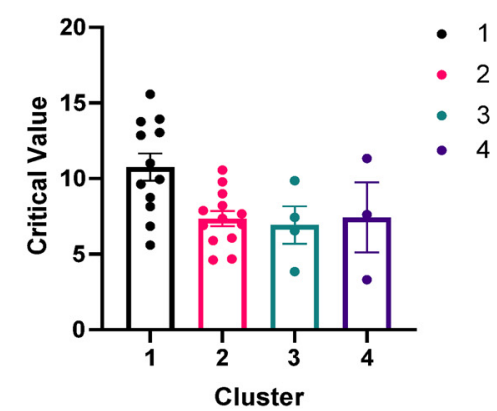

Dendritic angle ( $\left.{ }^{\circ}\right)$

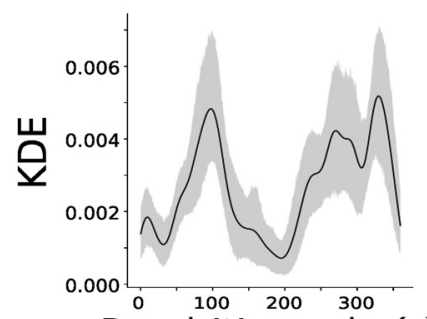

Dendritic angle ( $\left.{ }^{\circ}\right)$
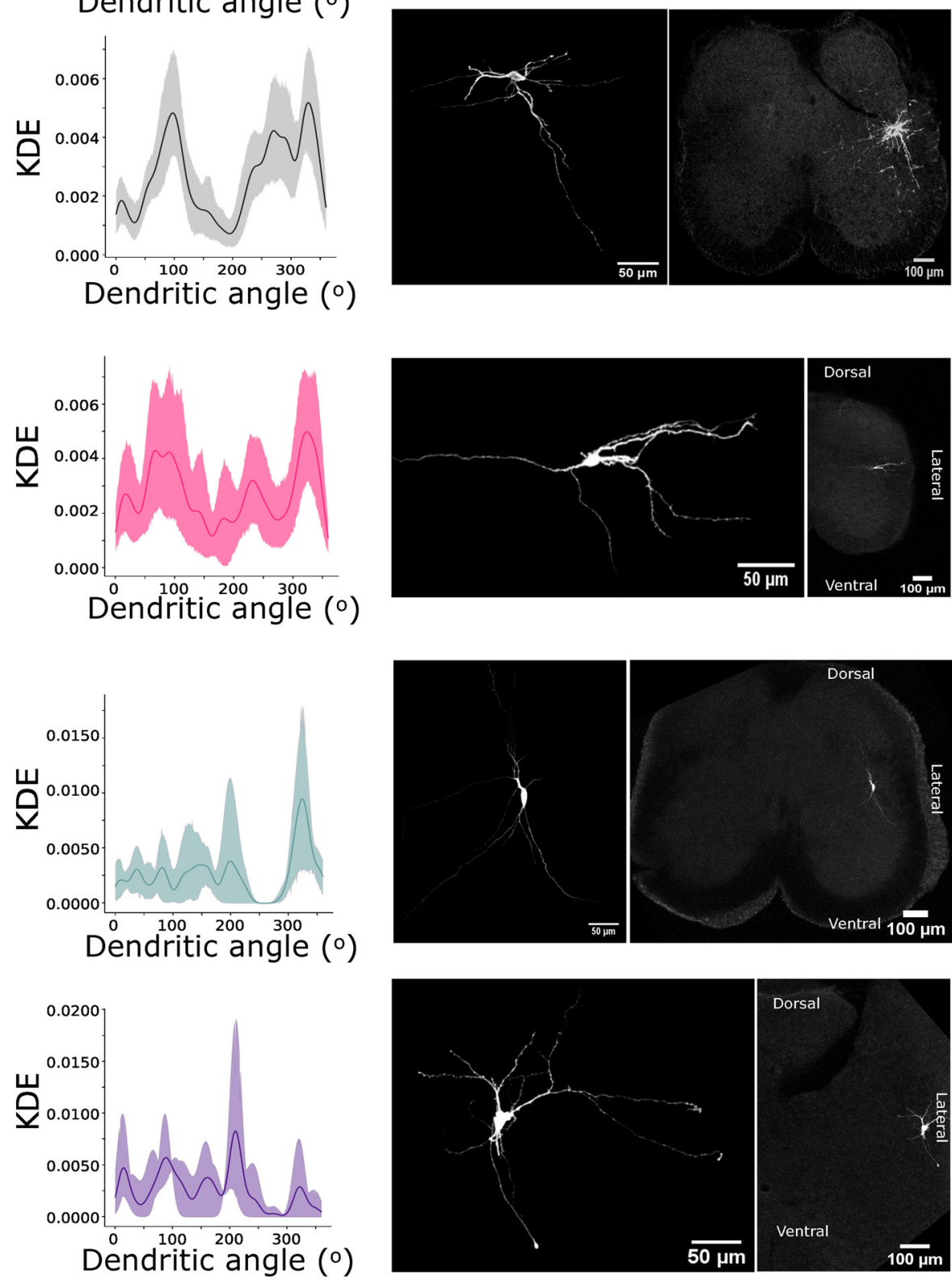

Figure 3. Distinct morphologies were associated with each e-type. $\boldsymbol{A}$, Comparison of the number of neurons with VRP neurites, typically associated with autonomic motoneurons. $\boldsymbol{B}$, Sholl analysis of reconstructed morphologies; ramification index and critical value between e-types. $\boldsymbol{C}$, Angles between the end of neurites and the soma were calculated to measure directional specificity $\left(^{\circ}\right)$ and spatial coverage $(\mu \mathrm{m})$. Note most e-types had a large ventro-lateral projection except for e-type 3. Left panel, Polar plots indicating average dendritic length within $45^{\circ}$ bins of dendritic end angle. Middle panel, Kernel density estimates (KDE) indicating the 
continued

directional likelihood of dendrites for each e-type. Right panel, Representative examples of reconstructed Neurobiotin-filled neurons for each e-type.

and constrain inhibitory input to putative PGN. Consequently, Kv3 channels may represent an important identifier of fast polysynaptic or recurrent inhibition of PGN.

\section{Discussion}

\section{The identity of e-types}

We identified four neuronal sub-populations in the lumbosacral intermediolateral spinal cord based on electrophysiological criteria; one type, e-type 3 , represented a fast-firing population and the remaining e-types, with longer AP duration and slower firing properties most likely represented parasympathetic motoneurons. We postulate that e-type 3 represented an interneuronal population for two main reasons: (1) we never observed a ventral root projecting neurite, typically indicative of PGN (Morgan, 2002a); and (2) e-type 3 neurons fired faster than reported frequencies for PGN (de Groat and Ryall, 1968a). Consequently, according to these criteria, e-types 1, 2 and 4 likely represent autonomic subpopulations of which multiple sub-types have indeed been postulated (Morgan et al., 1993). This is an important characterization that will improve our understanding of the parasympathetic function of the spinal cord, however it is important to note that the machine learning method of clustering used here is far from deterministic and represents a best estimate at the number and defining characteristics of subpopulations. However, by beginning to name specific neuronal types in this autonomic region, as has been accomplished in the dorsal horn (Todd, 2017), we can begin to ask what role they play in crucial physiological processes that involve parasympathetic input from the spinal cord, as is seen during the micturition reflex.

A
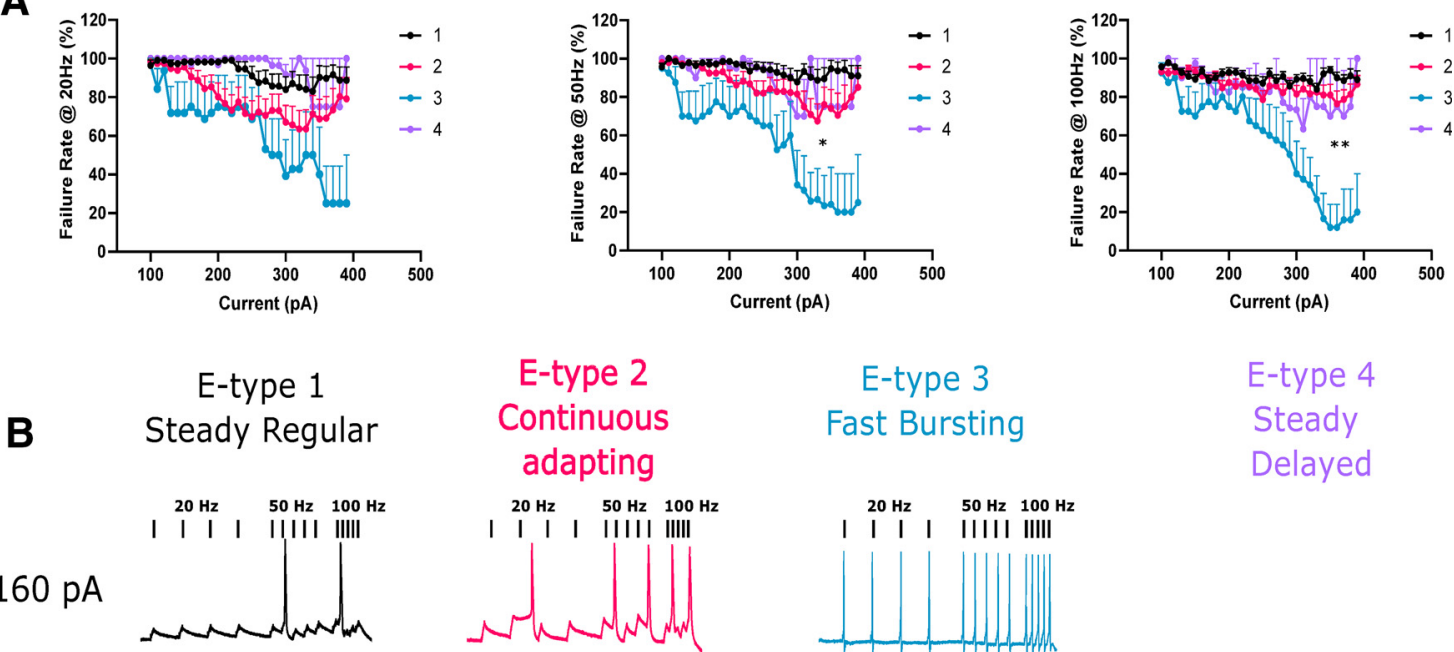

E-type 2

Continuous

E-type 3

Fast Bursting

E-type 4

Steady adapting
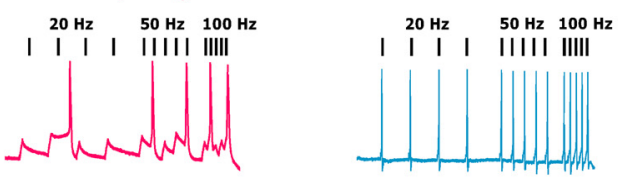

Delayed
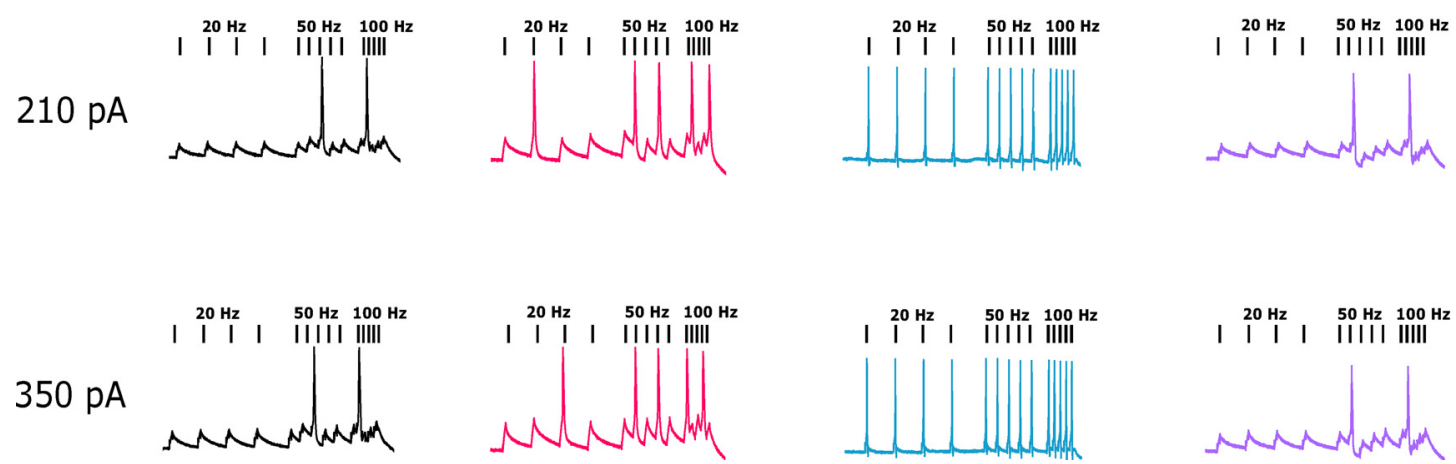

Figure 4. E-type 3 accurately followed high frequencies of stimulation. $\boldsymbol{A}, 10$-ms square current pulses were applied to neurons at different rates $(20,50$, and $100 \mathrm{~Hz})$ and at increasing amplitudes. Failure rate was quantified as a failure to fire an AP during a pulse. E-type 3 successfully followed faster stimulation frequencies significantly better than other e-types. $\boldsymbol{B}$, Representative examples of APs evoked at different stimulation frequencies and at different current amplitudes for each e-type. pA, picoAmps; Hz, Hertz. ${ }^{*} p<0.05$; ${ }^{\star *} p<0.01$. 
A
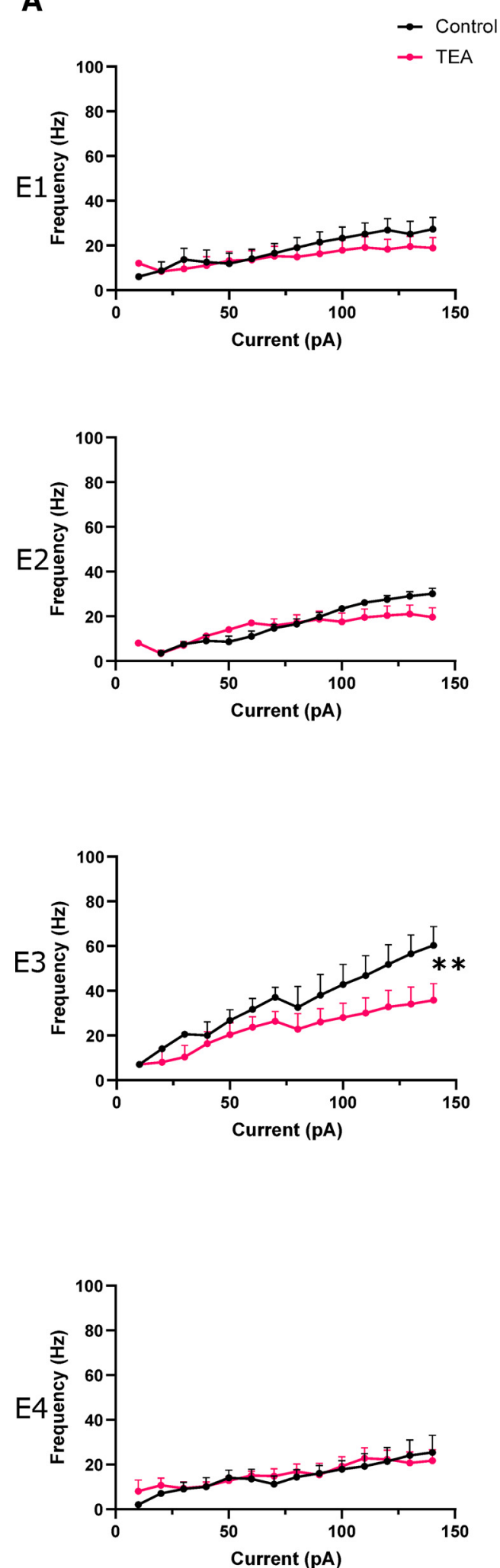
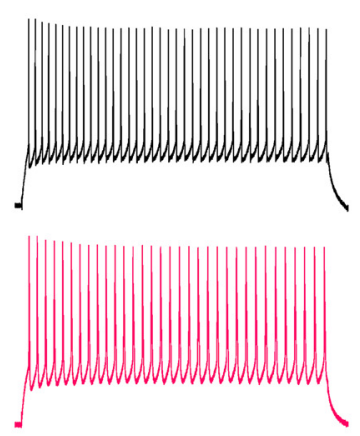

B
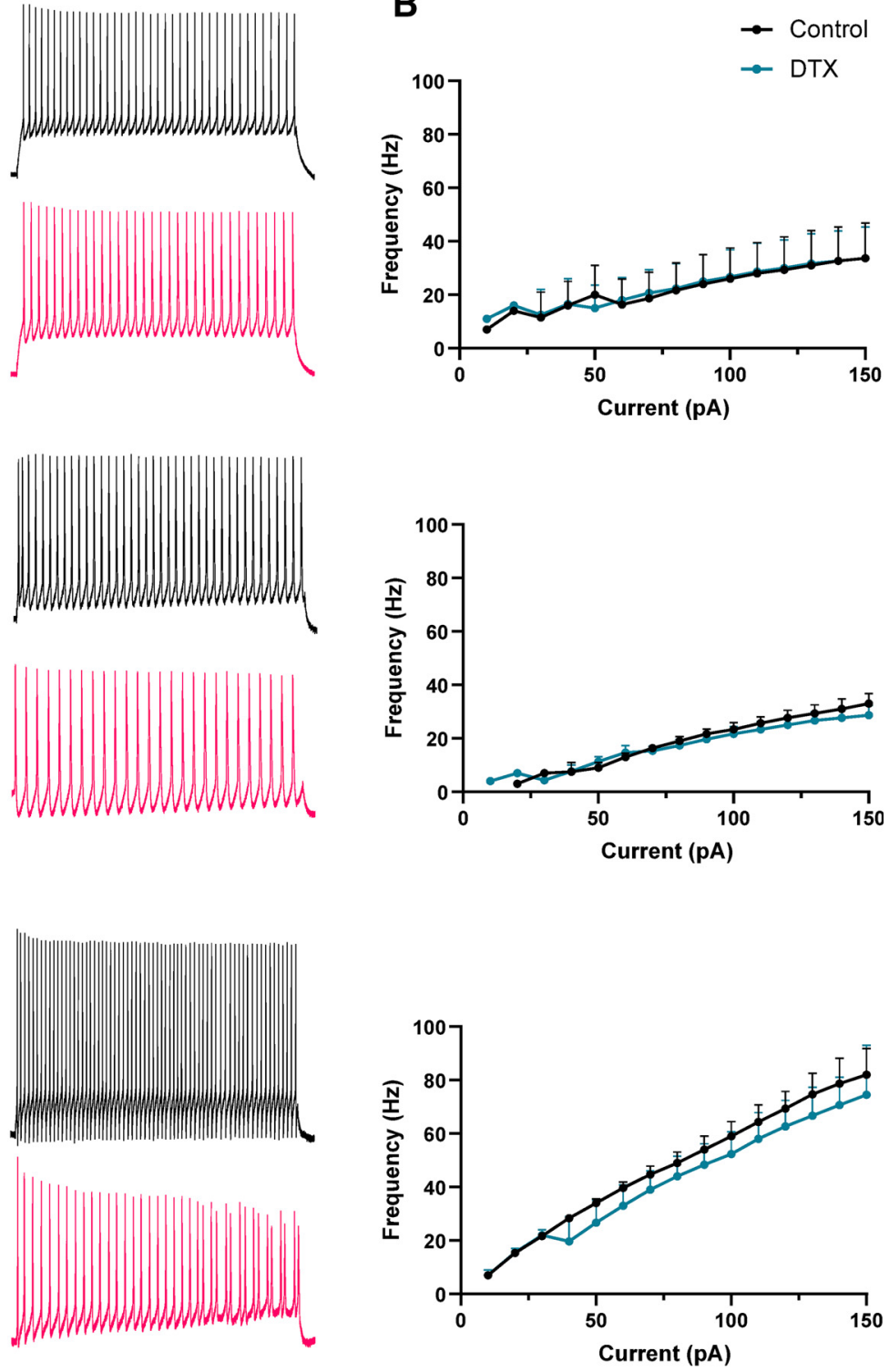

Figure 5. TEA impairs fast firing of e-type 3. $\boldsymbol{A}$, left panel, Firing frequency-current plots for each e-type (1-4) in control aCSF and in presence of $0.5 \mathrm{~mm}$ TEA. Note significant reduction in firing rate at $140 \mathrm{pA}$ in e-type 3. Right panel, Representative traces of neuronal firing in control aCSF and $0.5 \mathrm{~mm}$ TEA. $\boldsymbol{B}$, Firing frequency of each e-type over a range of current amplitudes in control aCSF and in presence of $10 \mathrm{~nm}$ DTX. pA, picoAmps; Hz, Hertz; TEA, tetraethylammonium chloride; DTX, dendrotoxin. ${ }^{\star \star} p<0.01$. 
A
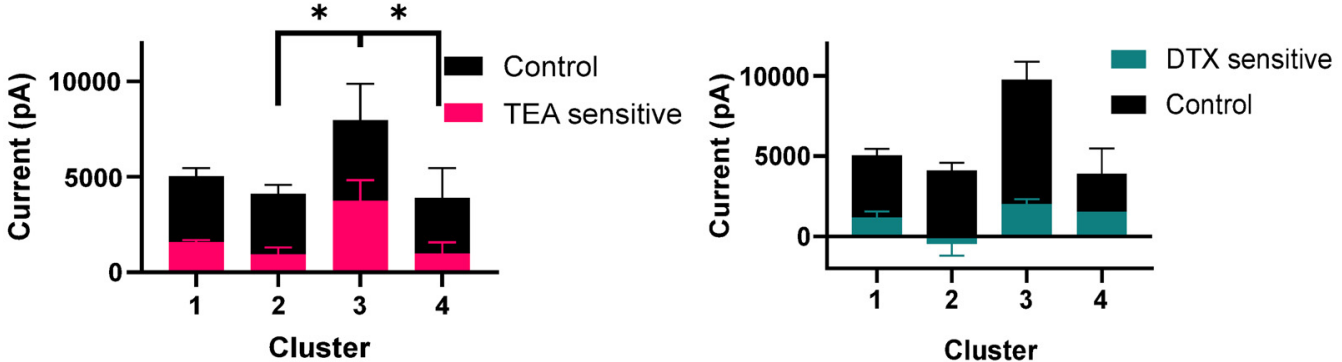

B
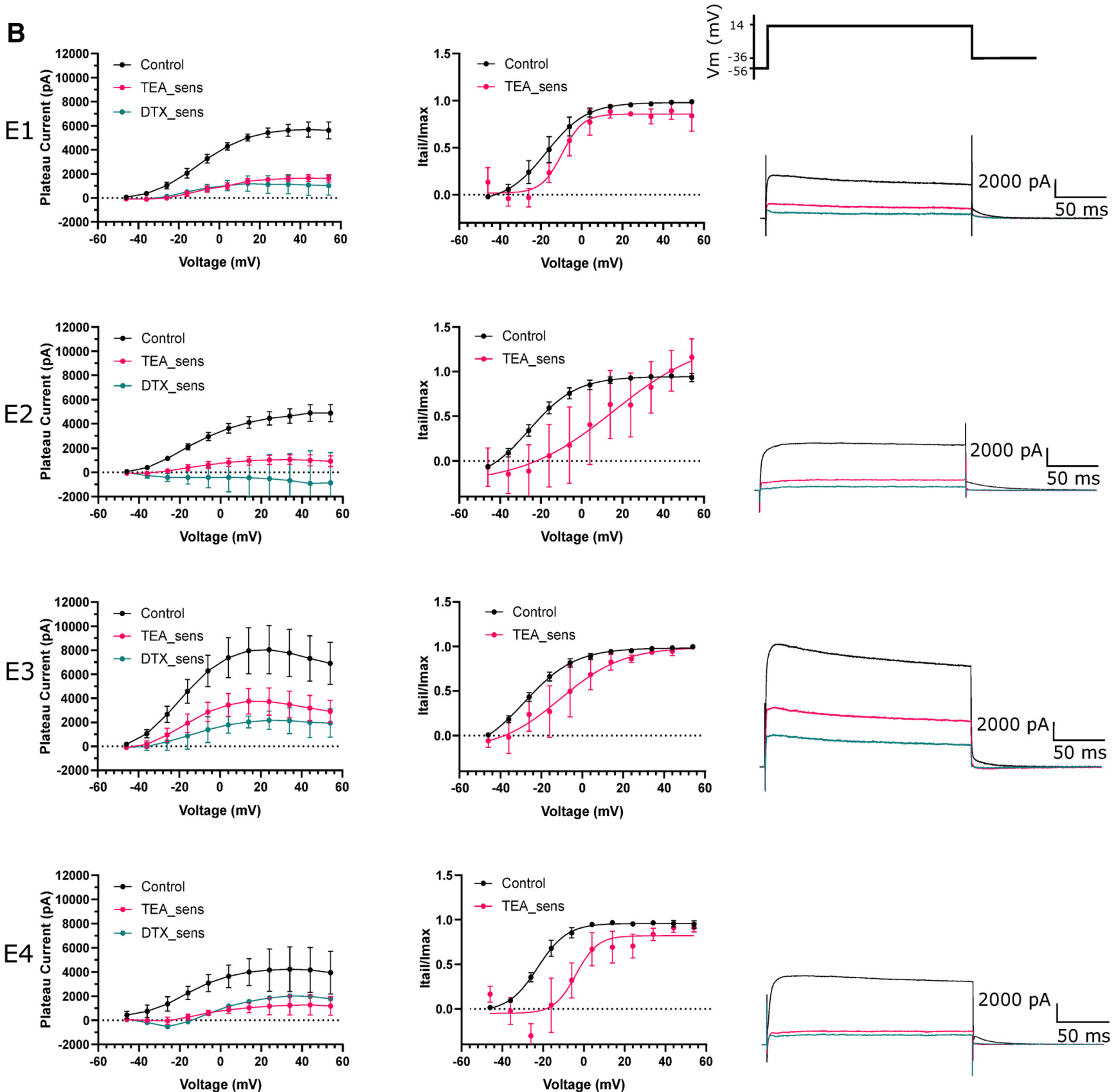

Figure 6. TEA-sensitive Kv currents greatly contribute to outward currents in e-type 3. $\boldsymbol{A}$, Plateau outward currents evoked during a voltage step to $14 \mathrm{mV}$ for each e-type in the presence of $0.5 \mathrm{~mm}$ TEA and $10 \mathrm{~nm}$ DTX. E-type 3 had significantly more TEA-sensitive current than e-type 1. B, left panel, Voltage-current plots for each e-type (1-4). TEA-sensitive and DTX-sensitive (TEA_sens, DTX_sens) currents were obtained by subtracting currents in TEA and DTX from currents in control aCSF, the residual revealing the currents blocked by each compound. Middle panel, Activation plots indicating current activation over voltage steps. Current was measured during deactivating 
continued

current tails and normalized to Imax. Right panel, Representative traces of outward currents evoked by a voltage step to $14 \mathrm{mV}$ for each e-type. E-type 3 sustained a larger total current and TEA-sensitive current. pA, picoAmps; mV, millivolts; TEA, tetraethylammonium chloride; DTX, dendrotoxin; sens, sensitive; Itail, tail current; Imax, maximum tail current. ${ }^{*} p<0.05$.

\section{Kv3 channels are crucial for the electrophysiological characteristics of e-type 3}

We further examined the biophysical underpinnings of the phenotype of e-type 3 with the hypothesis that expression of Kv3 channels underlie the fast-firing ability.
Kv3 channels are well known to confer rapid firing properties in multiple other CNS regions (Erisir et al., 1999; Lien and Jonas, 2003). Furthermore, association of the Kv3.1b subunit with intermediolateral fast-firing interneurons in the thoracic spinal cord has been previously established

A
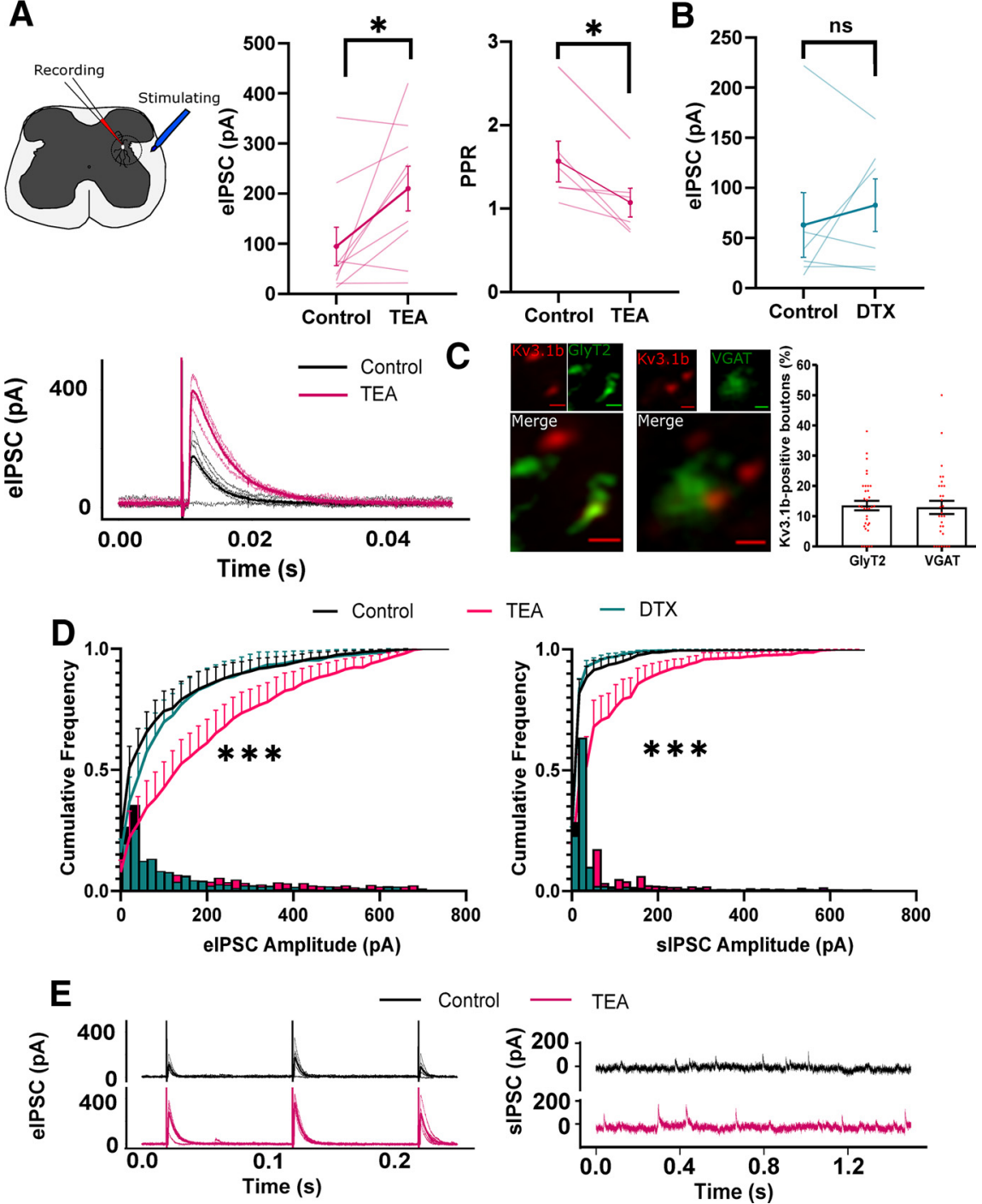

Figure 7. Kv3 blockade potentiates descending and local inhibitory synaptic responses. A bipolar electrode positioned in the lateral white matter was used to evoke descending synaptic currents. Neurons were held at $0 \mathrm{mV}$ to isolate IPSCs. $\boldsymbol{A}$, left panel, eIPSC amplitude between control and $0.5 \mathrm{~mm}$ TEA (each line represents an individual cell). Right panel, PPR between IPSCs $100 \mathrm{~ms}$ apart in $0.5 \mathrm{~mm}$ TEA. Bottom panel, Representative example of potentiated IPSC amplitude in TEA. B, Effect of bath application of 10 nM DTX on elPSC amplitude. C, left panel, Super-resolution Airyscan images showing clear overlap of example synaptic boutons with Kv3.1b IF with GlyT2 IF. right panel, Kv3.1b co-localization with inhibitory markers GlyT2 and VGAT represented as the number of Kv3.1b positive boutons. $\boldsymbol{D}$, Cumulative (line) and relative frequency (bar) plots of elPSC (left panel) and sIPSC (right panel) amplitudes in the presence of $0.5 \mathrm{~mm}$ TEA and $10 \mathrm{~nm}$ DTX. $\boldsymbol{E}$, Representative traces of elPSCs (left panel) and sIPSCs (right panel) in control and $0.5 \mathrm{~mm}$ TEA. pA, picoAmps; TEA, tetraethylammonium chloride; DTX, dendrotoxin; elPSC, evoked IPSC;sIPSC, spontaneous IPSC; GlyT2, glycine transporter 2; vGAT, vesicular GABA transporter; IF, immunofluorescence. ${ }^{*} p<0.05$; ${ }^{\star \star \star} p$ $<0.005$; ns- non-significant. 

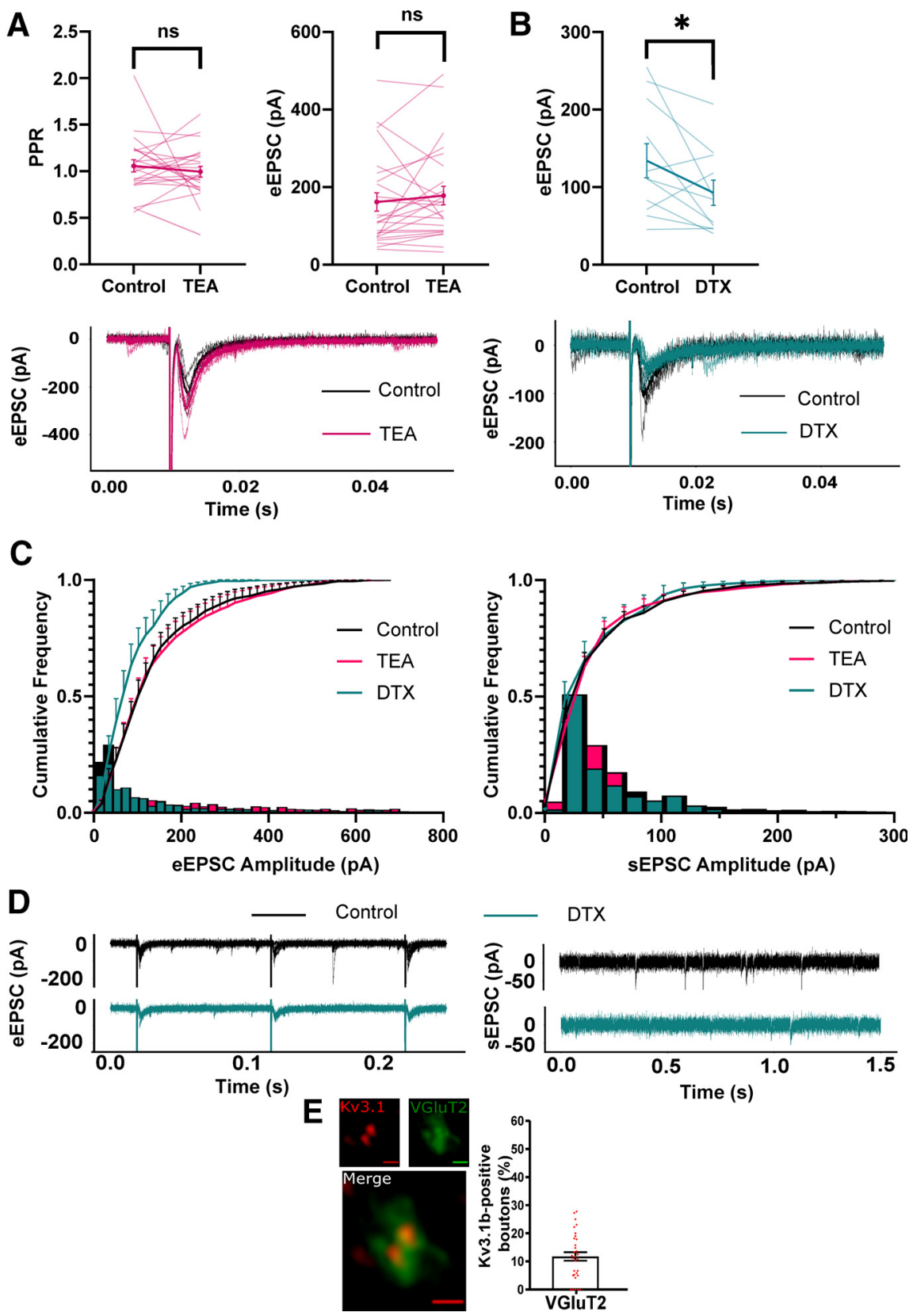

Figure 8. Kv3 blockade has no effect on excitatory synaptic responses. Neurons were held at $-56 \mathrm{mV}$ to isolate EPSCs. $\boldsymbol{A}$, Left panel, PPR between EPSCs 100ms apart in 0.5 mM TEA. Right panel, eEPSC amplitude between control and 0.5 mm TEA (each line represents an individual cell). Bottom panel, Representative example of unaffected EPSC amplitude in TEA. B, upper panel, Effect of bath application of $10 \mathrm{~nm}$ DTX on eEPSC amplitude. Lower panel, Representative example of eEPSC in control and DTX. C , Cumulative (line) and relative frequency (bar) plots of eEPSC (left panel) and sEPSC amplitudes in the presence of $0.5 \mathrm{~mm}$ TEA and 10 nм DTX. D, Representative traces of eEPSCs (left panel) and sEPSCs (right panel) in control and 10 nм DTX. E, Example Airyscan images and bar chart showing limited co-localization of VGluT2 boutons with Kv3.1b. pA, picoAmps; TEA, tetraethylammonium chloride; DTX, dendrotoxin; eEPSC, evoked EPSC; sEPSC, spontaneous EPSC; vGluT2, vesicular glutamate transporter 2; IF, immunofluorescence. ${ }^{*} p<0.05$.

(Deuchars et al., 2001; Brooke et al., 2002). In this study, application of Kv3 channel blocker, TEA, reduced firing frequency and isolated high-voltage activated outward currents that constituted a large proportion of total outward current. Furthermore, neuronal expression of Kv3 subunits in this area confirmed the importance of Kv3 channels in the physiology of this neuronal class. However, functionally distinguishing contributions of Kv3 subunits to the firing phenotype of e-type 3 is difficult without selective inhibitors for each subunit. This would require the use of knock-out Kv3 mouse lines in future experiments to evaluate the contribution of each subunit to 
the phenotype of e-type 3 as well as the effect on bladder control (Choudhury et al., 2020). Kv3 channels have been selectively associated with inhibitory populations in ventral and dorsal regions; Kv3 subunits are expressed in neurons that fire at high frequency such as Renshaw cells that inhibit motoneuron output (Song et al., 2006) and inhibitory dorsal horn interneurons that gate sensory input (Nowak et al., 2011). Are Kv3 channels also associated with inhibitory populations in the intermediolateral autonomic zone? Viral tracing studies show that interneurons synaptically connected to PGN are in the autonomic zone and focal extracellular stimulation of these local interneurons evoke both EPSCs and IPSCs in PGN (Araki, 1994; Araki and De Groat, 1996; Nadelhaft et al., 2000; de Groat et al., 2015). However, the TEA-sensitivity of local inhibitory but not excitatory inputs onto putative PGN suggests that Kv3 channels are associated with inhibitory populations synaptically connected to PGN. Our data did not distinguish an average difference in local and descending excitatory input amplitude after Kv3 blockade, but some examples are identifiable within our dataset that show potentiated excitatory synaptic responses. Further, Kv3.4 subunits have been associated with sensory glutamatergic afferent terminals in the dorsal horn (Muqeem et al., 2018); thus, we cannot completely rule out association of Kv3 channels with excitatory neurons. Indeed, our attempts to address this question directly using paired recordings within the intermediolateral region found limited connectivity, thus we could not distinguish between excitatory and inhibitory populations synaptically connected to PGN. The role of Kv3 channels at the synaptic terminal is well established, where application of Kv3 channel blockers broadens the presynaptic AP, increases $\mathrm{Ca}^{2+}$ influx and neurotransmitter release (Ishikawa et al., 2003; Goldberg et al., 2005; Kuznetsov et al., 2012; Rowan et al., 2014). Our findings further corroborate this role in the spinal cord, however, with a degree of selectivity for inhibitory terminals indicating that Kv3 channels are more likely to constrain inhibitory output. The punctate immunofluorescence of Kv3.1b and Kv3.3 suggests both subunits may be important in presynaptic dynamics. We observed no activity dependent facilitation of postsynaptic currents, but whether the inactivation of Kv3.3 is insufficient to elicit such a mechanism, as has been demonstrated for Kv3.4, is not known (Rowan and Christie, 2017).

\section{Other distinct ionic contributions to e-types}

We primarily focused on Kv3 channels in fast-firing neurons; however, there are clear indications of other ionic currents within our data. All e-types displayed a prominent voltage deflection "sag" characteristic of Ih during hyperpolarizing current injections, likely mediated by HCN channels. The delayed firing of e-type 4 is highly suggestive of IA typically conducted via Kv4 channels. Indeed, other autonomic neurons such as sympathetic preganglionic neurons have been found to express IA, where it endows low-pass filtering of incoming excitatory synaptic input by decreasing EPSP summation between 15 and $40 \mathrm{~Hz}$, thus reducing transmission of higher frequency activity (Briant et al., 2014). This was reflected in e-type 4 being unable to follow higher frequencies of stimuli and thus a similar low pass filter may exist in PGN. Voltage clamp data from all e-types also indicated a high-voltage activating delayed rectifier with slow activation that was TEA and DTX-insensitive often indicative of expression of Kv2 channels (Johnston et al., 2010). The slow kinetics of Kv2 channels ensure AP amplitudes are consistent by ensuring hyperpolarized interspike potentials and thus a stable pool of available non-inactivated $\mathrm{NaV}$ channels (Johnston et al., 2008). This ubiquitous expression likely explains the consistent AP amplitudes observed in all our etypes.

\section{An autonomic Renshaw cell?}

A fundamental question is what is the purpose for an inhibitory neuronal class to fire so much faster than its autonomic neighbors? A possible hypothesis is to enable rapid recurrent inhibition of PGNs to suppress activation of the parasympathetic motoneuronal pool and thus suppress contraction of the bladder detrusor smooth muscle. PGN extend axon collaterals within the spinal cord indicating a likely recurrent activation of other spinal neurons (Morgan et al., 1991; Morgan, 2002b). Furthermore, a recurrent inhibitory reflex akin to that of the motoneuronRenshaw cell motif has been described for PGNs (de Groat and Ryall, 1968b). This inhibition was shown to be glycinergic in nature, was mediated by fast-firing interneurons in the immediate vicinity of PGNs and depressed bladder detrusor contractions. The similar localization and phenotype of the e-type 3 class suggests that this neuronal population could represent a candidate "autonomic Renshaw cell." Indeed, previous research using rabies virus identified Kv3-expressing neurons antecedent to autonomic neurons in the thoracic spinal cord (Brooke et al., 2002). Furthermore, Kv3 channels consistently correlate with the fast-firing inhibitory element of recurrent inhibitory circuits, from Renshaw cells to cortical neurons (Song et al., 2006; Espinosa et al., 2008). Renshaw cells are thought to limit or terminate the activity of somatic motoneurons as well as potentially determine selective excitability of motoneurons (Noga et al., 1987). Analogously, an autonomic Renshaw cell, could completely suppress PGN activity and bladder contraction. However, this does not seem to be the case when the volume of the bladder is high (de Groat and Ryall, 1968b) but perhaps represents a mechanism of PGN quiescence during bladder filling and continence. Alternatively, with the expression of Ih current in putative PGN e-types, recurrent inhibition could define a rhythmic pattern of activity through rebound depolarization (van Hook and Berson, 2010), a pattern that could contribute to the spontaneous contractions observed for bladder detrusor muscle. Additionally, recurrent inhibition could allow selective activation of PGN with the greatest degree of excitation within the autonomic pool, where activity of the most excited PGN suppresses weakly excited PGN to better coordinate organs of the lower urinary tract during bladder voiding. These hypotheses assume multiple strong inhibitory inputs proximal to the soma to suppress PGN firing, however 
distal inputs may instead shunt dendritic afferent input as has been observed for Renshaw cells and motoneuron dendrites (Bhumbra et al., 2014). Kv3 channels have become pharmacological targets for a variety of neurologic disorders in the past decade, with novel compounds typically acting to slow the rate of firing of Kv3 expressing neurons or recover the firing rate in impaired paradigms (Rosato-Siri et al., 2015; Brown et al., 2016; Parekh et al., 2018). A pharmacologicallyinduced reduction in the rate of inhibition to PGN could confer increased parasympathetic activity and unwanted bladder detrusor contractions during bladder filling and typical periods of continence. However, there is some evidence of reductions of Kv3 subunits in the CNS during aging, and these may lead to impaired function of fast-firing neurons (Zettel et al., 2007). Perhaps firing-impaired interneurons in the spinal cord could disrupt spinal parasympathetic dynamics that correlate with age-related decline in bladder control and be amenable to pharmacological recovery. By fundamentally characterizing the individual neuron types within this circuitry and the biophysical mechanisms that underpin the phenotype of those cells, we can begin to understand and modulate spinal parasympathetic dynamics during important physiological reflexes such as the micturition reflex.

\section{References}

Araki I (1994) Inhibitory postsynaptic currents and the effects of GABA on visually identified sacral parasympathetic preganglionic neurons in neonatal rats. J Neurophysiol 72:2903-2910.

Araki I, De Groat WC (1996) Unitary excitatory synaptic currents in preganglionic neurons mediated by two distinct groups of interneurons in neonatal rat sacral parasympathetic nucleus. $J$ Neurophysiol 76:215-226.

Bhumbra GS, Bannatyne XBA, Watanabe M, Todd XAJ, Maxwell DJ, Beato XM (2014) The recurrent case for the Renshaw cell. J Neurosci 34:12919-12932.

Bolte S, Cordelieres FP (2006) A guided tour into subcellular colocalization analysis in light microscopy. J Microsc 224:213-232.

Briant LJB, Stalbovskiy AO, Nolan MF, Champneys AR, Pickering AE (2014) Increased intrinsic excitability of muscle vasoconstrictor preganglionic neurons may contribute to the elevated sympathetic activity in hypertensive rats. J Neurophysiol 112:2756-2778.

Brooke RE, Pyner S, Mcleish P, Buchan S, Deuchars J, Deuchars SA (2002) Spinal cord interneurones labelled transneuronally from the adrenal gland by a GFP-herpes virus construct contain the potassium channel subunit Kv3. 1b. Auton Neurosci 98:45-50.

Brooke RE, Atkinson L, Edwards I, Parson SH, Deuchars J (2006) Immunohistochemical localisation of the voltage gated potassium ion channel subunit Kv3. 3 in the rat medulla oblongata and thoracic spinal cord. Brain Res 1070:101-115.

Brown MR, El-hassar L, Zhang Y, Alvaro G, Large CH, Kaczmarek LK (2016) Physiological modulators of Kv3. 1 channels adjust firing patterns of auditory brain stem neurons. J Neurophysiol 116:106121.

Choudhury N, Linley D, Richardson A, Anderson M, Robinson SW, Marra V, Ciampani V, Walter SM, Kopp-Scheinpflug C, Steinert JR, Forsythe ID (2020) Kv3.1 and Kv3.3 subunits differentially contribute to Kv3 channels and action potential repolarization in principal neurons of the auditory brainstem. J Physiol 598:2199-2222.

Coetzee WA, Amarillo Y, Chiu J, Chow A, Lau D, McCormack T, Moreno H, Nadal MS, Ozaita A, Pountney D, Saganich M, VegaSaenz De Miera E, Rudy B (1999) Molecular diversity of K+ channels. Ann NY Acad Sci 868:233-255.

de Groat WC, Ryall RW (1968a) The identification and characteristics of sacral parasympathetic preganglionic neurones. J Physiol 196:563-577. de Groat WC, Ryall RW (1968b) Recurrent inhibition in sacral parasympathetic pathways to the bladder. J Physiol 196:579-591.

de Groat WC, Griffiths D, Yoshimura N (2015) Neural control of the lower urinary tract. Compr Physiol 5:327-396.

Deuchars SA, Brooke RE, Frater B, Deuchars J (2001) Properties of interneurones in the intermediolateral cell column of the rat spinal cord: role of the potassium channel subunit Kv3.1. Neuroscience 106:433-446.

Erisir A, Lau D, Rudy B, Leonard CS (1999) Function of specific K+ channels in sustained high-frequency firing of fast-spiking neocortical interneurons. J Neurophysiol 82:2476-2489.

Espinosa F, Torres-vega MA, Marks GA, Joho RH (2008) Ablation of Kv3. 1 and Kv3. 3 potassium channels disrupts thalamocortical oscillations in vitro and in vivo. J Neurosci 28:5570-5581.

Fowler CJ, Griffiths D, de Groat WC (2008) The neural control of micturition. Nat Rev Neurosci 9:453-466.

Goldberg EM, Watanabe S, Chang SY, Joho RH, Huang ZJ, Leonard CS, Rudy B (2005) Specific functions of synaptically localized potassium channels in synaptic transmission at the neocortical GABAergic fast-spiking cell synapse. J Neurosci 25:5230-5235.

Huma Z, Du Beau A, Brown C, Maxwell DJ (2014) Origin and neurochemical properties of bulbospinal neurons projecting to the rat lumbar spinal cord via the medial longitudinal fasciculus and caudal ventrolateral medulla. Front Neural Circuits 8:1-14.

Ishikawa T, Nakamura Y, Saitoh N, Li W-B, Iwasaki S, Takahashi T (2003) Distinct roles of Kv1 and Kv3 potassium channels at the calyx of held presynaptic terminal. J Neurosci 23:10445-10453.

Johnston J, Griffin SJ, Baker C, Skrzypiec A, Chernova T, Forsythe ID (2008) Initial segment Kv2.2 channels mediate a slow delayed rectifier and maintain high frequency action potential firing in medial nucleus of the trapezoid body neurons. J Physiol 586:34933509.

Johnston J, Forsythe ID, Kopp-Scheinpflug C (2010) Going native: voltage-gated potassium channels controlling neuronal excitability. J Physiol 588:3187-3200.

Jursky F, Nelson N (1995) Localization of glycine neurotransmitter transporter (GLYT2) reveals correlation with the distribution of glycine receptor. J Neurochem 64:1026-1033.

Kaczmarek LK, Zhang Y (2017) Kv3 channels: enablers of rapid firing, neurotransmitter release, and neuronal endurance. Physiol Rev 97:1431-1468.

Kuznetsov KI, Grygorov OO, Yu V, Veselovsky NS, Fedulova SA (2012) Cell Calcium K v 3 channels modulate calcium signals induced by fast firing patterns in the rat retinal ganglion cells. Cell Calcium 52:405-411.

Lien CC, Jonas P (2003) Kv3 potassium conductance is necessary and kinetically optimized for high-frequency action potential generation in hippocampal interneurons. J Neurosci 23:2058-2068.

Merolli A, Louro P, Kohn J (2019) Reciprocal nerve staining (RNS) for the concurrent detection of choline acetyltransferase and myelin basic protein on paraffin-embedded sections. J Neurosci Methods 311:235-238

Morgan C (2002a) Axons of sacral preganglionic neurons in the cat: I. Origin, initial segment, and myelination. J Neurocytol 30:523-544.

Morgan C (2002b) Axons of sacral preganglionic neurons in the cat: II. Axon collaterals. J Neurocytol 30:767-787.

Morgan C, Nadelhaft I, de Groat WC (1979) Location of bladder preganglionic neurons within the sacral parasympathetic nucleus of the cat. Neurosci Lett 14:189-194.

Morgan CW, de Groat WC, Felkins LA, Zhang SJ (1991) Axon collaterals indicate broad intraspinal role for sacral preganglionic neurons. Proc Natl Acad Sci USA 88:6888-6892.

Morgan CW, De Groat WC, Felkins LA, Zhang SJ (1993) Intracellular injection of neurobiotin or horseradish peroxidase reveals separate types of preganglionic neurons in the sacral parasympathetic nucleus of the cat. J Comp Neurol 331:161-182.

Muqeem T, Ghosh B, Pinto V, Lepore AC, Covarrubias M (2018) Regulation of nociceptive glutamatergic signaling by presynaptic Kv3.4 channels in the rat spinal dorsal horn. J Neurosci 38:37293740 . 
Nadelhaft I, Vera PL, Card JP, Miselis RR (2000) Central nervous system neurons labeled following the injection of pseudorabies virus into the rat prostate gland. Prostate 44:240-247.

Noga BR, Shefchyk SJ, Jamal J, Jordan LM (1987) The role of Renshaw cells in locomotion: antagonism of their excitation from motor axon collaterals with intravenous mecamylamine. Exp Brain Res 66:99-105.

Nowak A, Mathieson HR, Chapman RJ, Janzsó G, Yanagawa $Y$, Obata K, Szabo G, King AE (2011) Kv3. 1b and Kv3. 3 channel subunit expression in murine spinal dorsal horn GABAergic interneurones. J Chem Neuroanat 42:30-38.

Parekh PK, Sidor MM, Gillman A, Becker-krail D, Bettelini L, Arban R, Alvaro GS, Zambello E, Mutinelli C, Huang Y, Large CH, Mcclung CA (2018) Antimanic efficacy of a novel Kv3 potassium channel modulator. Neuropsychopharmacology 43:435-444.

Rosato-Siri MD, Zambello E, Mutinelli C, Garbati N, Benedetti R, Aldegheri L, Graziani F, Virginio C, Alvaro G, Large CH (2015) A novel modulator of $\mathrm{Kv} 3$ potassium channels regulates the firing of parvalbumin-positive cortical interneurons. J Pharmacol Exp Ther 354:251-260.

Rowan MJM, Christie JM (2017) Rapid state-dependent alteration in Kv3 channel availability drives flexible synaptic signaling dependent on somatic subthreshold depolarization. Cell Rep 18:20182029.

Rowan MJM, Tranquil E, Christie JM (2014) Distinct Kv channel subtypes contribute to differences in spike signaling properties in the axon initial segment and presynaptic boutons of cerebellar interneurons. J Neurosci 34:6611-6623.

Rudy B, Mcbain CJ (2001) Kv3 channels: voltage-gated K+ channels designed for high-frequency repetitive firing. Trends Neurosci 24:517-526.
Shefchyk SJ (2001) Sacral spinal interneurones and the control of urinary bladder and urethral striated sphincter muscle function. $\mathrm{J}$ Physiol 533:57-63.

Soares D, Goldrick I, Lemon RN, Kraskov A, Greensmith L, Kalmar B (2017) Expression of Kv3.1b potassium channel is widespread in macaque motor cortex pyramidal cells: a histological comparison between rat and macaque. J Comp Neurol 525:2164-2174.

Song Z, Hu J, Rudy B, Redman SJ (2006) Developmental changes in the expression of calbindin and potassium-channel subunits Kv3. $1 \mathrm{~b}$ and Kv3. 2. Neuroscience 139:531-538.

Todd AJ (2017) Identifying functional populations among the interneurons in laminae I-III of the spinal dorsal horn. Mol Pain 13:1744806917693003.

Tozuka Y, Fukuda S, Namba T, Seki T, Hisatsune T (2005) GABAergic excitation promotes neuronal differentiation in adult hippocampal progenitor cells. Neuron 47:803-815.

van Hook MJ, Berson DM (2010) Hyperpolarization-activated current $(/ \mathrm{h})$ in ganglion-cell photoreceptors. PLoS One 5:e15344.

Weiser M, Bueno E, Sekirnjak C, Martone ME, Baker H, Hillman D, Chen S, Thornhill W, Ellisman M, Rudy B (1995) The potassium channel subunit KV3.1b is localized to somatic and axonal membranes of specific populations of CNS. J Neurosci 15:4298-4314.

Zettel ML, Zhu X, O'Neill WE, Frisina RD (2007) Age-related decline in Kv3.1b expression in the mouse auditory brainstem correlates with functional deficits in the medial olivocochlear efferent system. J Assoc Res Otolaryngol 8:280-293.

Zhu F, Cizeron M, Qu Z, Benavides-Piccione R, Kopanitsa MV, Skene NG, Koniaris B, DeFelipe J, Fransén E, Komiyama NH, Grant SGN (2018) Architecture of the mouse brain synaptome. Neuron 99:781-799.e10. 